\title{
Vehicle Detection and Counting to Identify Traffic Density in The Intersection of Road Using Image Processing
}

\author{
Fitria Claudya Lahinta ${ }^{1}$, Zahir Zainuddin ${ }^{2}$, Syafruddin Syarif ${ }^{3}$ \\ ${ }^{1}$ Department of Electrical Engineering, Faculty of Engineering, Universitas Hasanuddin \\ Makassar, Indonesia. 92119 \\ ${ }^{2,3}$ Department of Electrical Engineering, Faculty of Engineering, Universitas Hasanuddin \\ Makassar, Indonesia. 92119 \\ \{lahintafc17d@student.unhas.ac.id ${ }^{1}$,zahir@unhas.ac.id², syafruddin.s@eng.unhas.ac.id ${ }^{3}$ \}
}

\begin{abstract}
Vehicle density information for traffic regulation including the timing of traffic lights is still very minimal. This study aims to calculate the number of vehicles at an intersection then classify the density level of each road segment. The detection process begins with taking video from Teling intersection of Manado City, Indonesia. Video processed using the Gaussian Mixture Model (GMM) algorithm and Morphological Operation (MO) to detect vehicles object in the form of BLOB (Binary Large Object). The results of the feature extraction are calculated to get the number of vehicles from the specified Region of Interest (ROI). The results of counting vehicles are classified according to the density level to be able to compare the level of congestion on each road segment. The results of the proposed system accuracy is $90.9 \%$ for the calculation of vehicles on the road. This research is expected to be implemented in Smart Traffic Light.
\end{abstract}

Keywords: Gaussian Mixture Model (GMM), Morphological Operation (MO), Vehicle Detection, Counting Vehicle, Teling Intersection.

\section{Introduction}

Based on data from the BPS North Sulawesi, economic growth in North Sulawesi up to 2017 increased by $6.32 \%$ [1]. Increased economic growth increases the number of transportation in North Sulawesi, while the development of infrastructure in North Sulawesi is still slow [2]. Along with the increase in number of vehicles, traffic light arrangements are needed in the management of traffic flows. But on the other side, the traffic lights make traffic jams worse because the timing conditions are still conventional. The timing conditions sometimes make a traffic jam on one road or several roads [3].

With the development of information and communication technology, researchers developed intelligent traffic lights, namely by utilizing the Advanced Traffic Management System (ATMS) technology. ATMS is information and communication technology as an alternative to vehicle density problems [4]. In Indonesia, Amirullah et al. designed a traffic control system using the fuzzy logic method. Fuzzy logic is applied to a system to reduce vehicle density by determining the membership function taken based on the calculation of the number of vehicles [5]. Meanwhile, in India, Dubey et al. control traffic lights with the integration of 
Internet of Things (IoT) with local conditions can be monitored by the Office were to control traffic lights [6].

In this paper, the author will design a system for calculating vehicles and classifying the number of vehicles using image processing techniques at intersections. Several related studies to measure vehicle density that had previously been carried out by Ghazal et al. who designed a PIC microcontroller based system. This system evaluates the density of vehicles using IR sensors. IR sensors are placed on one road which is the density limit of one road segment [7]. In addition to the use of sensors to measure density, video data is also effective for use in detecting the density of vehicles on roads. Basri et al. used the Gaussian Mixture Model method by analyzing Region of Interest (ROI), BLOB analysis and frame jump techniques to detect cars and motorcycles. The technical optimization offered got a $29 \%$ increase in car detection and a $20.47 \%$ decrease for motorcycles [8]. Further research by Ihtisham Ali et al. which implemented an Intelligent Traffic Management System (EtrafficWarden) using Image Processing with the Control Algorithm. Videos are taken from the built-in operation provided by OpenCV. Videos are taken from the camera and process frame by frame with the process continues until the user chooses to stop it [9].

Based on data obtained in the field during the pre-study for seven days, the condition of the queue of vehicles at the intersection varies in density on each side of the road in terms of time. Those results in the timing of the traffic not being too effective to be used on the road. Besides the waiting time that is not very effective traffic lights results in saturation of the driver of the vehicle, especially those located at Jln. Toar and Jln. Lumimuut which has uphill lanes. Determining the location of the camera also affects the frame to be taken because of the road conditions mentioned. This study uses the Gaussian Mixture Model and Morphology Operation method for vehicle detection. The results of the number of vehicle queues are expected to be classified according to the type of density in this case divided into three classification parts, namely light, medium and solid. The results of the classification are expected to be integrated into traffic lights with traffic light timing parameters.

\section{Materials and Method}

In this section, the method for identifying and calculating vehicles is explained. Gaussian Mixture Model is used for detecting background and foreground on video files. The foreground is processed again using Morphological Operation to eliminate noise and optimise vehicle detection. Then the results of the Morphological Operation process will be segmented to get the Large Object Bit (BLOB). The blob amount of the frame is counted to get the number of vehicles. The following explained about the method used.

\subsection{Video Input}

Vehicle detection begins with video input with a resolution of 640x480 from the Teling intersection of Manado City, North Sulawesi, Indonesia. The camera used is the type of Yoosen IP Camera. The camera is placed at the height of 5 meters with a slope of 55 degrees. The location of the camera at the intersection can be seen in Figure 1. 


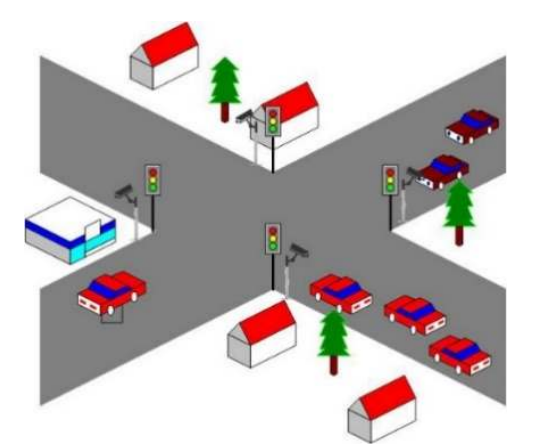

Fig. 1. CCTV location at the intersection

Cameras placed on each side of the road at the intersection. Video input from one road segment will be compared each frame to get the background and foreground. The basic technique for detecting changes from frame $\mathrm{f}\left(\mathrm{x}, \mathrm{y}, t_{l}\right)$ and frame $\left.\mathrm{f}\left(\mathrm{x}, \mathrm{y}, t_{i-1}\right)\right)$ where at time $t_{l}$ and at time $t_{i-1}$ produces a value not equal to zero [10].

\subsection{Preprocessing}

In this study, two stages are carried out in the preprocessing section. The first step is to change RGB colour frames to grayscale. Grayscale is the intensity of pixels in the black range (0) to white (255). The transformation of RBG to grayscale aims to flatten the pixel value of RGB [11]. The next preprocessing process is a gaussian blur; the gaussian blur aims to reduce image noise.

\subsection{Segmentation}

The next step after preprocessing is segmentation. Segmentation aims to separate the object (foreground) from the background. The method used in the segmentation process is the Gaussian Mixture Model. GMM with the master estimates a good solution distribution in the search space as a set of clusters, assuming that a good solution in each cluster follows a Gaussian distribution [12]. In the Gaussian Mixture Model, each pixel in a frame is modelled into a Gaussian distribution. Its intensity distinguishes each pixel in RGB (Red, Green, Blue). Then each pixel is calculated as the foreground (FG) or background (BG) value using equation (1).

$$
P\left(X_{t}\right)=\sum_{i=1}^{K} \omega_{i, t} \cdot n\left(X_{t}, \mu_{k}, \Sigma_{i, t}\right)
$$

Where $\mathrm{P}\left(\mathrm{X}_{\mathrm{t}}\right)$ is the probability for pixels $(\mathrm{x}, \mathrm{y})$ at time $\mathrm{t} . \mathrm{K}$ is the most distributed component or model used. $\omega_{\mathrm{i}, \mathrm{t}}$ is the weight for distribution to $\mathrm{k}$ at frame $\mathrm{t} . \mu_{\mathrm{k}}$ is the mean from distribution to $\mathrm{k}$ at frame t. $\Sigma_{\mathrm{i}, \mathrm{t}}$ is a covariance matrix [13].

The next step in the segmentation section is the threshold. At the threshold process, a limit value is given for pixels. The threshold value used in this study is 25 . The intensity value that is more than or equal to the threshold value will be changed to 1 (white) with an intensity value less than the threshold value will be changed to 0 (black) so that the output from the threshold is a binary image [14]. The threshold method equation can be calculated from the following equation (2). 


$$
g=(x, y)=\left\{\begin{array}{l}
1, f(x, y) \geq 25 \\
0, f(x, y)<25
\end{array}\right.
$$

\subsection{Morphological Operation}

Morphological operations are image processing techniques that aim to change the shape of the original image object. Morphological operations involve two-pixel arrays. The first array is a morphologically processed image and the second array is the kernel [15].

In this paper optimisation of vehicle object detection uses three types of morphological operation processes. The morphological process used is closing, opening and dilation. Closing is a morphological process that is preceded by a dilation process and then an erosion process. Dilation is a technique to enlarge an object segment from an image by adding layers around the object. For example, there is a background value of 0 that is adjacent to an object that has a value of 1 , then a background point of 0 is changed to 1 . Erosion is a technique to reduce or erode the edges of objects. For example, there is a point value of 1 that is neighbouring with a background value of 0 , then the point of the object that value of 1 is changed to 0 . The closing process aims to close the hole in the object's vehicle. The closing technique equation can be seen in the following equation (3).

$$
\text { Closing }=\mathrm{A} \cdot \mathrm{B}=(\mathrm{A} \oplus \mathrm{B}) \ominus \mathrm{B}
$$

Next to the opening technique, the erosion process will be carried out first and followed by a dilation process. Opening is the opposite of the closing process. The method of opening the vehicle detection aims to soften the edges of the object [16]. The equation for the opening technique can be seen in the following equation (4).

$$
\text { Opening }=\mathrm{A} \circ \mathrm{B}=(\mathrm{A} \ominus \mathrm{B}) \oplus \mathrm{B}
$$

The last morphological process used in this study is dilation. This technique is used so that objects can look more prominent and clearer. The equation of the dilation technique can be seen in the following equation.

$$
\text { Dilation }=\mathrm{A} \oplus \mathrm{B}
$$

\subsection{Blob Analysis}

BLOB analysis is a method used to determine a collection of pixels that are related to each other. The image must be processed with the threshold method first to determine the colour above and below the threshold value. The image above the threshold value can be obtained as a group or an object that is needed. Objects in two or three-dimensional shapes are usually called BLOB [17]. 


\section{Results and Discussion}

The process of detecting vehicle objects that are started on preprocessing as described initially can be seen in the following figure 2 .

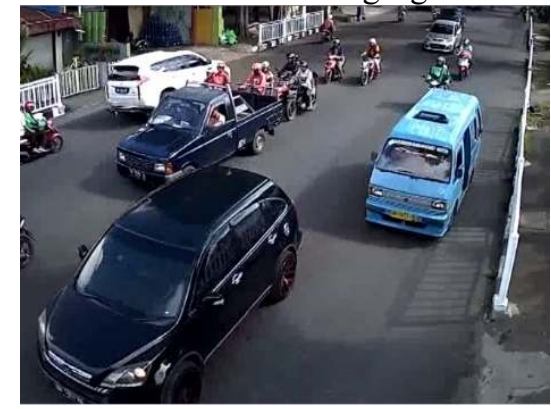

(a)

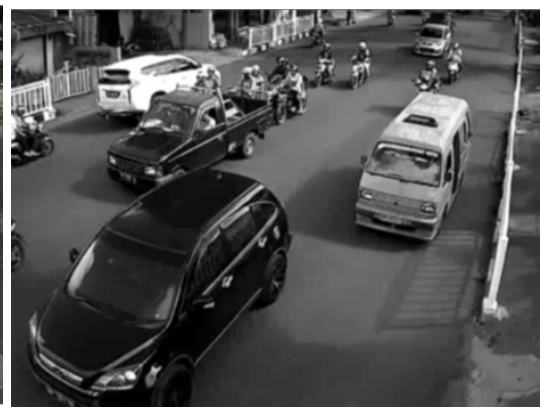

(b)

Fig. 2. Preprocessing Vehicle Detection : (a) real video, (b) video after preprocessing

The preprocessing process in this paper is the transformation of RGB images to Grayscale to get a range of values 0 - 255 (grey scale) and then a gaussian blur to eliminate noise in grayscale images. Furthermore, in the segmentation process using the GMM algorithm, the parameter $\alpha$ value that is good located between $0.05-0.5$. For an $\alpha$ value of less than 0.05 , it cannot update the model quickly. While the value for $\alpha$ is more than 0.5 , the foreground and background cannot be separated. Also, there is a determination of the threshold value in the segmentation process. The threshold value obtained is 25 . The following in Figure 3 is the result of segmentation.

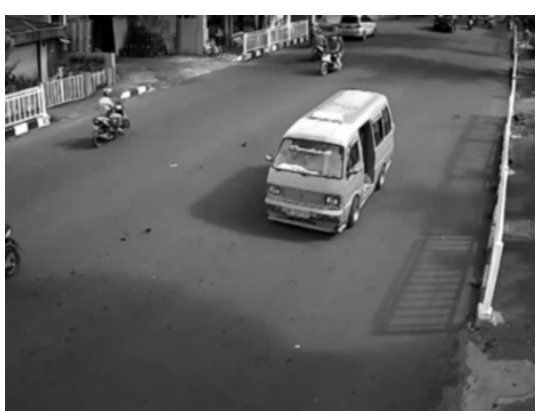

(a)

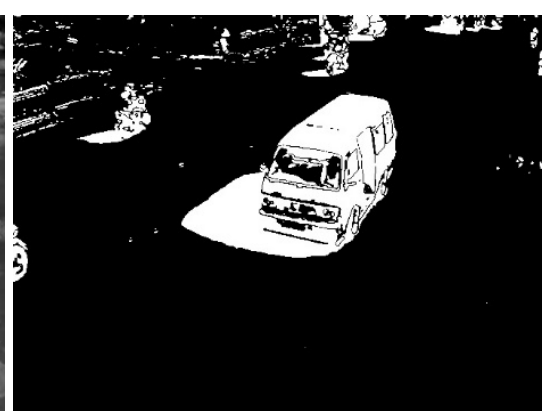

(b)

Fig. 3. Segmentation process using GMM : (a) Grayscale Frame, (b) GMM Process 
In the morphological operation stage, vehicle objects are detected as foreground will be optimised to get a better shape. As explained earlier, three processes will be carried out in the phase of morphological operation. The three methods are closing, opening and dilation. In the three morphological processes, the kernel used is $(5,5)$. The following in Figure 4 is the result of closing and opening process.

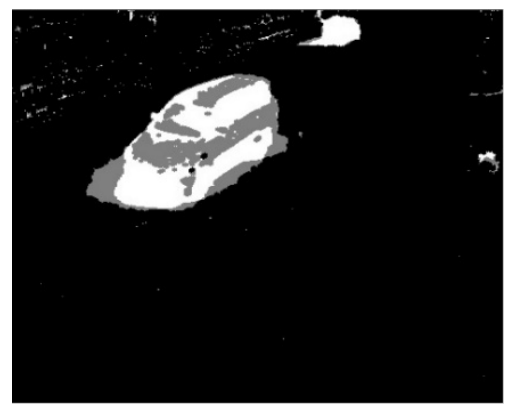

(a)

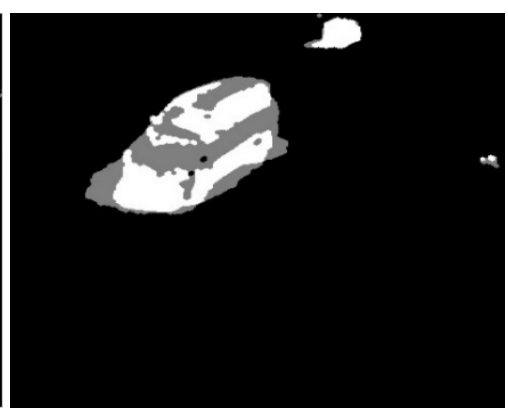

(b)

Fig. 4. Morfologi Operation : (a) Closing, (b) Opening

In the opening process, the results of the image look smoother, and the noise on the edges is also lost. To enlarge an object, a layer is added around the object using a dilation process. The kernel that is carried out in the dilation process is the same as the kernel which is carried out during the closing and opening process, kernel $(5,5)$. The results of the dilation process can be seen in the following figure 5.

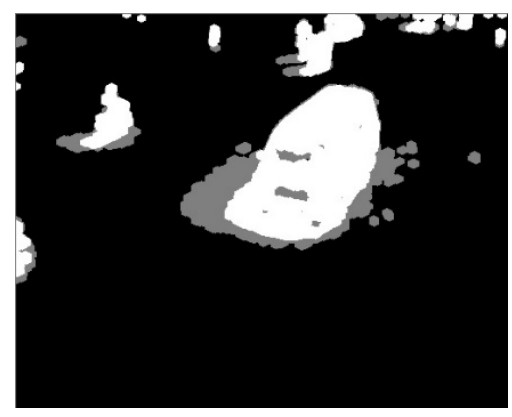

Fig. 5. Dilation

The results of the dilation process of the vehicle object have been enlarged, but the shadow of the vehicle is still detected. This can reduce the accuracy of the next process for counted vehicles because the shadows of the vehicle can be connected with other vehicles. As a result, the vehicle calculation process is not effective because there are two or more vehicles, but only one vehicle is counted. Therefore the threshold is carried out again in the last process to reduce the shadow on the vehicle. The threshold value obtained in this process is 220 . The result of adding a threshold value can be seen in the following figure 6 . 


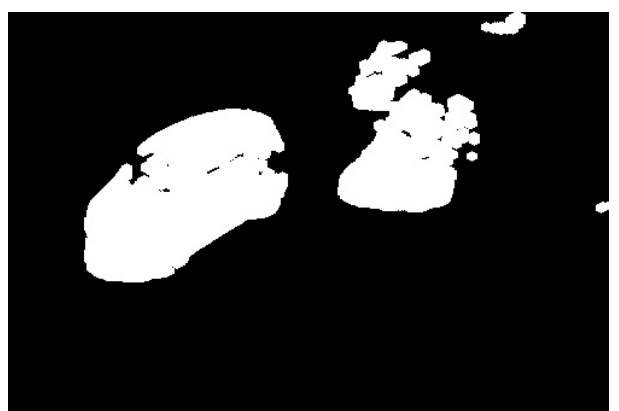

Fig. 6. Threshold after dilation process

Furthermore, the BLOB analysis gave a limit to the number of object contours. In this study, the contour is limited to 500-5000. This contour restriction area is quite large because the calculation will be done if the vehicle passes the ROI (Region of Interest), and the ROI position is at the end when the vehicle approaches the camera so that the vehicle that is getting closer to the camera will look bigger. The vehicle calculation process starts when the vehicle passes the specified ROI. The results can be seen in the following figure 7.

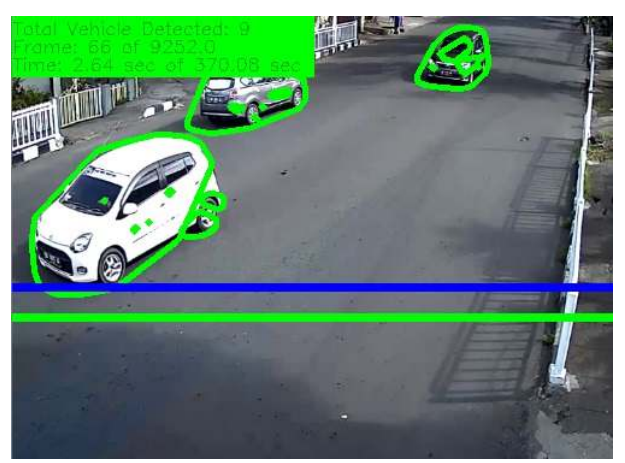

Fig. 7. Design System

ROI (Region of Interest) gave a limit by a line where when the vehicle passes through the area; the vehicle calculation process will begin. The result can be seen in the following figure 8 .

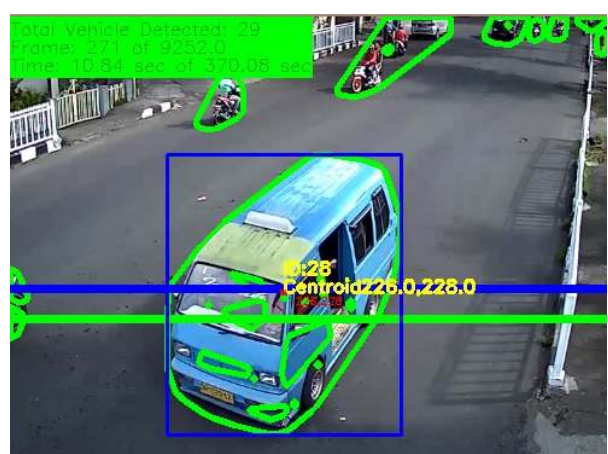

Fig. 8. Vehicle counting process 
After the calculation process is carried out the classification process, in this paper the classification is divided based on the number of cars. The results of the classification can be seen in the following figure 9.

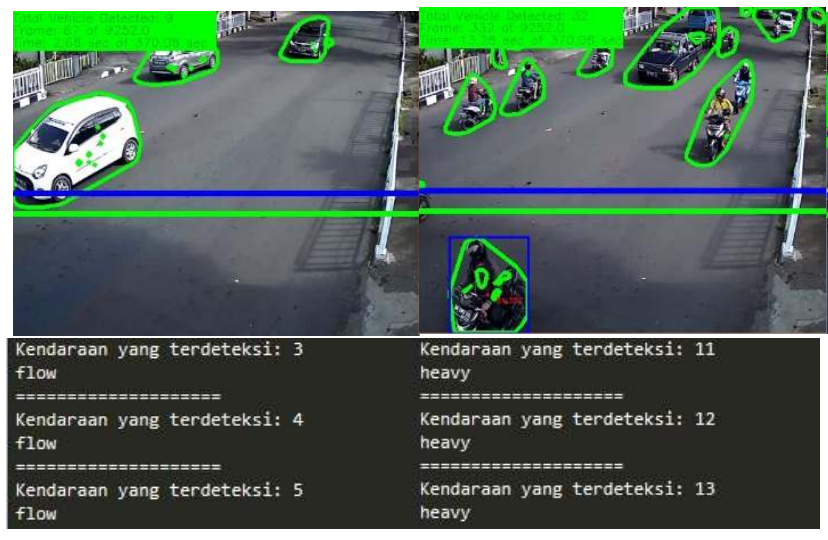

(a) Flow Condition

(b) Heavy Condition

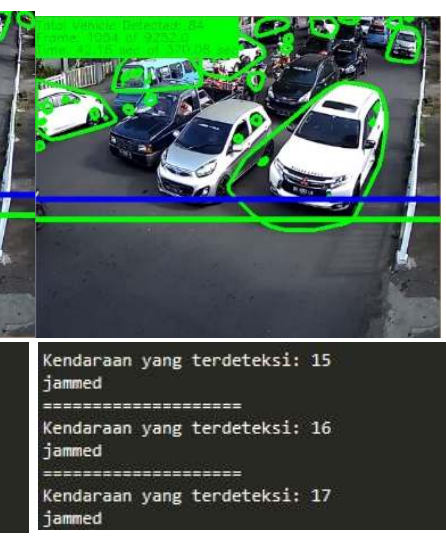

(c) Jammed Condition

Fig. 9. System testing result

Comparison between system testing result and manual computation results can be seen in the following table 1 .

Table 1. Vehicle Calculation Result

\begin{tabular}{cccc}
\hline Data & Actual & System & Accuracy \\
\hline 1 & 10 & 9 & $90 \%$ \\
2 & 15 & 13 & $86 \%$ \\
3 & 20 & 22 & $90 \%$ \\
4 & 22 & 19 & $86 \%$ \\
5 & 30 & 32 & $90 \%$ \\
6 & 28 & 25 & $89 \%$ \\
7 & 24 & 23 & $95 \%$ \\
8 & 19 & 19 & $100 \%$ \\
9 & 15 & 17 & $88 \%$ \\
10 & 20 & 19 & $95 \%$ \\
AVG & & & $\mathbf{9 0 . 9 \%}$ \\
\hline
\end{tabular}




\section{Conclusion}

This study used video data taken at the Teling intersection, Manado City, North Sulawesi. The position of the camera is very important to get a good video to process into the system created. The camera's height is 5 meters, and the slope angle is 55 degrees. The vehicle detection process begins with the GMM algorithm which aims to get the results of segmentation in the form of objects from the vehicle. Furthermore, the results of this study object from the vehicle carried out the morphological process to get better object results and can remove the shadow from the vehicle. The shadow of the vehicle can intergrated two or more vehicles so that the vehicle which is more than one is only counted by one vehicle. Therefore, in this study morphological processes are very important to do. The test results by comparing manual observations using the system got an accuracy of $90.9 \%$. The results of this study are expected to be integrated into the traffic so that the light control system can be balanced with the density of each road segment.

\section{References}

[1] "Badan Pusat Statistik Provinsi Sulawesi Utara." [Online]. Available: https://sulut.bps.go.id/quickMap.html. [Accessed: 28-Jul-2018].

[2] "Jumlah Kendaraan Terus Meningkat, Kadishub Manado Ingatkan Pengguna Jalan Suluttoday.com." .

[3] R. E. P. Polah, R. Sengkey, and Y. D. Y. Rindengan, "Perancangan Simulasi Otomatis Traffic light Menggunakan Citra Digital Studi Kasus Persimpangan Toar-Lumimuut," J. Tek. Elektro Dan Komput., vol. 4, no. 4, pp. 35-45, Aug. (2015).

[4] Indrabayu, I. Nurtanio, and F. Mayasari, "PROTOTIPE SISTEM TRAFFICLIGHT CERDAS UNTUK SOLUSI KEMACETAN LALU LINTAS (Sistem Transportasi Cerdas Selaras Kultur-Sosial Benua Maritim Indonesia),” (2015).

[5] I. Amirullah, Y. Lesmana, A. A. Ilham, I. Nurtanio, and S. Hamid, "An intelligent traffic light system for reducing number of queuing cars in complex road junction," ICIC Express Lett. Part B Appl., vol. 8, pp. 999-1006, Jun. (2017).

[6] A. Dubey, M. Lakhani, S. Dave, and J. J. Patoliya, "Internet of Things based adaptive traffic management system as a part of Intelligent Transportation System (ITS)," in 2017 International Conference on Soft Computing and its Engineering Applications (icSoftComp), pp. 1-6, doi : 10.1109/ICSOFTCOMP.2017.8280081, (2016).

[7] B. Ghazal, K. ElKhatib, K. Chahine, and M. Kherfan, "Smart traffic light control system," in 2016 Third International Conference on Electrical, Electronics, Computer Engineering and their Applications (EECEA), pp. 140-145, doi : 10.1109/EECEA.2016.7470780, (2016).

[8] Basri, Indrabayu, and A. Achmad, "Gaussian Mixture Models optimization for counting the numbers of vehicle by adjusting the Region of Interest under heavy traffic condition," in 2015 International Seminar on Intelligent Technology and Its Applications (ISITIA), pp. 245-250, doi : 10.1109/ISITIA.2015.7219986, (2015)

[9] I. Ali, A. Malik, W. Ahmed, and S. A. Khan, "Real-Time Vehicle Recognition and Improved Traffic Congestion Resolution," in 2015 13th International Conference on Frontiers of Information Technology (FIT), pp. 228-233, doi : 10.1109/FIT.2015.48, (2015).

[10] Wisnu Jatmiko, "Integrated Traffic Intelligent System." [Online]. Available: https://www.researchgate.net/publication/305769217_Integrated_Traffic_Intelligent_Sy stem. [Accessed: 14-Aug-2018]. 
[11] Z. A. V, B. Kurian, and N. Rajesh, "Multiple license plate recognition for intelligent traffic management," in 2011 Annual IEEE India Conference, pp. 1-4, doi : 10.1109/INDCON.2011.6139402, (2011)..

[12] Syafruddin Syarif, Syafaruddin, Wardi, and Shinya Kobayashi, "QUALITY ANALYSIS AND ILLEGAL LOGGING IMAGE DETECTION USING SYARITAR METHOD." Int. J. Innov., vol. 11, no. 3, pp. 1093-1099, (2015)

[13] D. A. Reynolds, "Gaussian Mixture Models," in Encyclopedia of Biometrics, (2009).

[14] Rafael C. Gonzalez and Richard E. Woods, Digital Image Processing. .

[15] A. Ajmal and I. M. Hussain, "Vehicle detection using morphological image processing technique," in 2010 International Conference on Multimedia Computing and Information Technology (MCIT), pp. 65-68, doi : 10.1109/MCIT.2010.5444851, (2010).

[16] Jun-Wei Hsieh and Yung-Sheng Chen, "Morphology-based license plate detection from complex scenes," in Object recognition supported by user interaction for service robots, 2002, vol. 3, pp. 176-179 vol.3, doi : 10.1109/ICPR.2002.1047823, (2002)

[17] E. Atkoc `iu, "Image Processing in Road Traffic Analysis," p. 18. 